\title{
Quadriceps Muscle Strength and Body Mass Index are Associated with Estimates of Physical Activity Postheart Transplantation
}

\author{
Rebecca L. Kelly, BPhty ${ }^{1,3,6}$, James R. Walsh, PhD $^{1,3,6}$, Jennifer D. Paratz, PhD ${ }^{2,3,4}$, \\ Stephanie T. Yerkovich, $\mathrm{PhD}^{2,3,4}$, Scott C. McKenzie, MBBS ${ }^{2,6}$, Norman R. Morris, $\mathrm{PhD}^{3,4,5}$
}

${ }^{1}$ Physiotherapy Department, The Prince Charles Hospital, Brisbane, Australia

${ }^{2}$ School of Medicine, The University of Queensland, Brisbane, Australia

${ }^{3}$ School of Allied Health Sciences, Griffith University, Gold Coast, Australia

${ }^{4}$ Menzies Health Institute, Griffith University, Gold Coast, Australia

${ }^{5}$ The Prince Charles Hospital Allied Health Research Collaborative. Queensland, Australia

${ }^{6}$ Advanced Heart Failure and Cardiac Transplant Unit, The Prince Charles Hospital,

Brisbane, Australia

\section{Correspondence:}

Rebecca Louise Kelly

Physiotherapy Department

The Prince Charles Hospital

Rode Rd

Chermside, 4032

Queensland

Australia

Telephone number: +61731394443

Email address: francis_becky@hotmail.com

Rebecca.Kelly2@health.qld.gov.au 


\section{Authorship}

Rebecca L Kelly ${ }^{1,3,6}$ BPhty: Participated in research design, writing the paper, performance of the research and data analysis.

Dr James R Walsh ${ }^{1,3,6} \mathrm{PhD}$ : Participated in research design, writing the paper and data analysis.

Dr Jennifer D Paratz ${ }^{2,3,4} \mathrm{PhD}$ : Participated in writing the paper.

Dr Stephanie T Yerkovich ${ }^{2} \mathrm{PhD}$ : Participated in writing the paper and data analysis.

Dr Scott C McKenzie ${ }^{2,6}$ MBBS: Participated in research design and writing the paper.

Professor Norman R Morris ${ }^{3,4,5} \mathrm{PhD}$ : Participated in research design, writing the paper and data analysis.

\section{Disclosure}

The authors declare no conflicts of interest.

\section{Funding}

The Prince Charles Hospital Foundation New Investigator Grant (NR2013-229) was utilized to assist with conducting this research. Amount awarded A\$9498.64 


\section{Abbreviations}

BMI, body mass index

CAV, cardiac allograft vasculopathy

$\mathrm{CR}$, cardiac rehabilitation

CVD, cardiovascular disease

EE, energy expenditure

$\mathrm{HF}$, heart failure

HTx, heart transplantation

LVEF, left ventricular ejection fraction

LTx, lung transplant

METs, metabolic equivalents

PA, physical activity

PAL, physical activity level

QS\%, quadriceps muscle strength corrected for body weight

QoL, quality of life

6MWD, 6 minute walk distance

6MWT, 6 minute walk test

SD, standard deviation

SWA, SenseWear Pro3 armband 
BACKGROUND: Whilst exercise capacity improves postheart transplantation (HTx), it remains unclear if the level of physical activity (PA) shows similar improvement. The purpose of this study was to (1) describe PA levels and (2) identify factors which may be associated with levels of PA post-HTx.

METHODS: A prospective observational cross-sectional study was conducted at a single centre HTx outpatient clinic. Medically stable adult recipients $\geq 6$ months post-HTx were recruited. Physical activity level (PAL) and average daily time spent at least moderately active ( $\geq 3$ metabolic equivalents (METs)) were estimated using a multisensor device. Factors investigated were demographic (age, sex, body mass index (BMI), time post-HTx and reason for HTx), corticosteroid use, exercise capacity (6 minute walk distance) and quadriceps muscle strength corrected for body weight (QS\%).

RESULTS: The mean post-HTx time of the 75 participants was $9.2 \pm 7.0 \mathrm{yrs}(0.5-26 \mathrm{yrs})$. Twenty-seven (36\%) were classified as extremely inactive (PAL<1.40); 26 (34.6\%) sedentary $(1.40 \leq \mathrm{PAL} \leq 1.69)$ and $22(29.3 \%)$ active $(\mathrm{PAL} \geq 1.70)$. Multivariable analysis showed greater QS\% $(\beta=0.004(0.002-0.006) \mathrm{p}=0.001)$ to be independently associated with increased PAL. For increased time $\geq 3 \mathrm{MET}$ s both greater $\mathrm{QS} \%(\beta=0.0164(0.003-0.029), \mathrm{p}=0.014)$ and lower BMI $(\beta=-0.0626(-0.115--0.0099), p=0.021)$ were independently associated.

CONCLUSIONS: The degree of observed sedentary behaviour post-HTx is surprising, with the majority of participants not reaching levels of PA recommended for health benefits. QS\% and BMI were the only factors found to be independently associated with estimates of PA. Further quality trials are required to demonstrate the long-term benefits of regular PA and investigate ways of increasing adherence to PA post-HTx. 


\section{INTRODUCTION}

Heart transplantation $(\mathrm{HTx})$ is a well-accepted treatment for end-stage heart failure(HF) ${ }^{1}$. Whilst survival rates in the first year post-HTx have improved; long-term survival has remained essentially unchanged for the last 20 years $^{2}$. Cardiovascular (CVD) events are a leading cause of death post-HTx ${ }^{3}$, with cardiac allograft vasculopathy (CAV) affecting 50\% of recipients by 10 years post-HTx ${ }^{4}$.

Obesity (Body mass index $(\mathrm{BMI}) \geq 30 \mathrm{~kg} / \mathrm{m}^{2}$ ) is common post- $\mathrm{HTx}{ }^{5,6}$ with recipients reported to gain approximately $10 \mathrm{~kg} 1$ year post-HTx ${ }^{6}$. Obesity post-HTx is associated with increased risk of $\mathrm{CVD}^{5,7}, \mathrm{CAV}^{7}$, rejection ${ }^{8,9}$ and diabetes ${ }^{7,9}$. The impact of obesity and physical inactivity on all-cause mortality in the general population is well described ${ }^{10,11}$. In healthy and chronic disease populations, increased exercise capacity and higher levels of physical activity (PA) have been found to have a favourable effect on CVD risk factors including blood pressure, diabetes and obesity ${ }^{12}$. Whilst the impact of low levels of PA has been reasonably well-documented in healthy and chronic heart disease populations ${ }^{13-16}$, this has not been well-described in the post-HTx population. There is evidence to suggest that exercise capacity improves post-HTx ${ }^{17-21}$. Studies have demonstrated the benefits of exercise training post-HTx, with exercise training reportedly improving long-term survival ${ }^{22}$ and peak oxygen consumption $^{23-25}$ and resistance training improving skeletal muscle mass ${ }^{26}$, strength $^{26}$ and bone density ${ }^{27}$. Andersen et al (2017) in a recent Cochrane review reported that exercisebased cardiac rehabilitation (CR) improves exercise capacity compared with no exercise post$\mathrm{HTx}^{21}$, yet they concluded that further adequately powered and high quality trials are needed to demonstrate the longer-term benefits of exercise post-HTx ${ }^{21}$. 
PA is defined as any bodily movement produced by skeletal muscles during every day functioning ${ }^{28}$, reflecting both the physical and behavioural performance of the individual ${ }^{29}$. Initiating and maintaining involvement in regular PA is determined by many variables ${ }^{30}$ including exercise-associated symptoms, peripheral muscle strength, training and disease status, mood, past behaviours, health beliefs, personality characteristics and socio-economic, cultural and external factors ${ }^{28,29,31,32}$. There are a variety of ways of measuring levels of PA, but multisensor devices that provide an estimate of energy expenditure (EE) normalised for resting EE offer several advantages ${ }^{33}$. In particular, using this approach, the physical activity level (PAL) can be derived. This measure, which takes into account differences in body size, expresses daily $\mathrm{EE}$ as a function of overnight $\mathrm{EE}^{34,35}$. Leading health agencies, including the World Health Organisation, have developed 3 broad PAL classes to categorise PA: extremely inactive (PAL $<1.40)^{33}$, sedentary (PAL 1.40-1.69) ${ }^{36}$ and active (PAL $\left.\geq 1.70\right)^{36}$. Healthy individuals who are extremely inactive (PAL <1.40) and sedentary (PAL 1.40-1.69) have been shown to have increased risk factors for CVD with those who are extremely inactive $(\mathrm{PAL}<1.40)$ at increased risk of CVD, diabetes and some types of cancer ${ }^{11,37}$. The mean PAL range for adults aged 18-64years in western populations has been documented as approximately 1.60 to 1.75 (sedentary to moderately active) ${ }^{33}$.

Only a few small studies have attempted to describe PA levels in the post-HTx population ${ }^{38-}$ 40. A greater body of evidence exists for the postlung transplant (LTx) population, with studies reporting reduced time spent walking and reduced time spent moderately active $(\geq 3 \mathrm{METs})^{41-44}$, with higher levels of daily PA related to greater exercise capacity, physical functioning and preserved muscle strength ${ }^{42}$. Whilst it is likely that similar factors are related to levels of PA in the HTx population, to date no study has reported the normalised PAL in a large group of HTx recipients using a reliable multisensor device or investigated factors that 
may be associated with estimates of PA. Therefore, the purpose of this study was to (1) describe PA levels post-HTx using a multisensor device (SenseWear Pro3 armband (SWA)) and (2) identify factors which may be associated with estimates of PA (PAL and average daily time spent at least moderately active ( $\geq 3$ METs)) post-HTx. We hypothesize that PAL and average daily time spent $\geq 3$ METs will correlate directly with 6 minute walk distance (6MWD) and quadriceps muscle strength corrected for body weight (QS\%), inversely with age, BMI, time post-HTx and ischaemic cardiomyopathy and no correlation with sex or other aetiologies of cardiomyopathy. 


\section{MATERIALS AND METHODS}

\section{Patient selection}

In this prospective observational cross-sectional study, medically stable adult HTx recipients were recruited from a single centre HTx outpatient clinic (The Prince Charles Hospital, Brisbane, Australia). Inclusion criteria were participants $\geq 6$ months post-HTx and able to wear the SWA $\geq 22$ hours/day for $\geq 3$ days. Exclusion criteria were left ventricular ejection fraction (LVEF) (by any imaging modality), less than 50\%, a rejection episode within the past 2 months or any injury or illness that could be reasonably expected to reduce mobility. This study was approved by the institutional ethics committee (HREC/13/QPCH/34) and informed written consent was gained from each participant.

\section{Study parameters}

Primary outcome measures of PAL and average daily time spent $\geq 3$ METs were estimated using the SWA. Participants were asked to wear the SWA continuously for 1 week except when participating in water-based activities ${ }^{45}$. The SWA has been validated against other techniques measuring EE including indirect calorimetry and the doubly labelled water method in healthy ${ }^{46,47}$ and chronic disease ${ }^{48}$ populations. Output parameters include PAL, average daily time spent $\geq 3$ METs, steps and time spent lying down and sleeping. Based on PAL, participants were classified as: extremely inactive $(\mathrm{PAL}<1.40)^{33}$, sedentary (PAL 1.40$1.69)^{36}$ or active $(\mathrm{PAL} \geq 1.70)^{36}$. Note whilst the SWA reports daily steps (also an indicator of PA), there is some evidence in other clinical populations that the device is inaccurate at detecting steps, particularly at slow walking speeds ${ }^{49}$. Hence, we did not report daily steps for this study. 


\section{Measures}

\section{Demographics}

Age, sex, height, body weight, BMI, time post-HTx, reason for HTx (aetiology of cardiomyopathy), immunosuppression regime at time of assessment and most recent postHTx LVEF within 12 months of assessment time point were recorded.

\section{Quadriceps muscle strength}

Quadriceps muscle strength was measured using a handheld dynamometer (Lafayette Manual Muscle Test System) as per published protocol $^{50}$ with an adjustable strap secured behind the participant's leg and held to the dynamometer to ensure an isometric contraction as previously described $^{51}$. In a seated position on a plinth with 90 degrees hip flexion, feet not touching the floor and the knee positioned at 70 degrees flexion, the participant was instructed to extend their knee maximally for 4 seconds with the peak force of the isometric contraction recorded in kilograms $(\mathrm{kg})$. Three attempts were conducted on each leg alternating between legs each time with at least 1 minute rest between attempts as per the published protocol $^{52}$. To standardise for body weight, quadriceps muscle strength was expressed as a percentage (QS\%) by adding together the best attempt on each leg and dividing by the participant's body weight $(\mathrm{kg})$ as per Walsh et al $(2014)^{51}$.

\section{Six minute walk test}

Exercise capacity was measured using the 6-minute walk test (6MWT) conducted on a thirty metre track in a hospital corridor as per standardised protocol ${ }^{53}$. Time was monitored using a stopwatch and participants were instructed to walk at their own pace whilst attempting to 
cover the greatest distance possible in the 6 minutes. Standard encouragement was provided every minute as per recommended guidelines ${ }^{53}$.

\section{Study protocol}

Following recruitment, participants performed the quadriceps strength test, 6MWT and had the SWA fitted on their right upper arm. Instructions were given to participants to wear the SWA in their home and community environment for 1 week. Upon return of the SWA the PA measures were uploaded and PAL and average daily time spent $\geq 3$ METs were estimated and recorded. These assessment measures are clinically relevant and easily reproducible.

\section{Statistical analysis}

Statistical analysis was performed using linear regression and spearman correlation as appropriate to determine predictors of PAL and average daily time spent $\geq 3 \mathrm{METs}$. Data was $\log$ transformed where it did not comply with normality. Univariable $(\mathrm{p}<0.1)$ and multivariable analysis was conducted with demographics and routine clinical measures thought likely to impact PAL and average daily time spent $\geq 3$ METs analysed. Variables included were age, sex, time post-HTx, BMI, reason for HTx (aetiology of cardiomyopathy), corticosteroid use at time of assessment, 6MWD and QS\%. Data is expressed as mean \pm SD unless otherwise stated.

\section{RESULTS}

\section{Participants}

Eighty-seven HTx recipients were recruited between February 2013 and August 2014. Of these, 9 were excluded due to wear time of the SWA device <22hrs/day for <3days, 1 for reduced LVEF (42\%) and 2 for device failure. Therefore, seventy-five participants, (20 
female, $26.7 \%$ ) and time post-HTx $9.2 \pm 7.0$ years were included (Table 1). Reasons for HTx were $27(35.5 \%)$ ischemic cardiomyopathy, 28 (37.3\%) idiopathic dilated cardiomyopathy and $20(26.3 \%)$ other (restrictive cardiomyopathy, familial, fabrys disease, hypertrophicobstructive cardiomyopathy, amyloid, congenital heart disease, sarcoidosis and valvular heart disease).

\section{Immunosuppression}

All participants were on an immunosuppression regimen including a calcineurin inhibitor and at least 1 agent from a different class. The majority of recipients were on a combination of cyclosporin $(n=55)$ and mycophenolate mofetil $(M M F)(n=36)$. Other immunosuppression medications used were tacrolimus $(n=18)$, everolimus $(n=11)$, sirolimus $(n=6)$, myfortic $(n=7)$ and azathioprine $(n=18)$. Most participants were not taking any oral corticosteroids (prednisolone) at the time of the study (65\%) with approximately 35\% taking at least $1 \mathrm{mg}$ per day (1 -10 mg) (Table 2).

\section{Outcomes}

The PA parameters are included in Table 3. The mean PAL for the group was $1.66 \pm 0.47$ and the mean average daily time spent $\geq 3$ METs was $143 \pm 155$ minutes. Using the PAL categories, $25(33.3 \%)$ participants were classified as extremely inactive (PAL <1.40), 28 (37.3\%) sedentary (PAL 1.40-1.69) and 22 (29.3\%) active (PAL $\geq 1.70)$. There was a similar distribution of participants on oral corticosteroids (prednisolone) at time of assessment in the physically active $(\mathrm{PAL} \geq 1.7)$ and inactive $(\mathrm{PAL}<1.7)$ groups (Table 2$)$. When we looked at corticosteriod use as a categorical value, we found no difference in PAL between those on prednisolone or not $(\mathrm{p}=0.60)$. When we looked at corticosteroid dose as a continuous variable, no difference was found between PAL and daily dose $(\mathrm{r}=-0.07, \mathrm{p}=0.56)$. When we 
compared demographic data, clinical measures (6MWD, QS\% and LVEF) and corticosteroid use of the physically active (PAL $\geq 1.7)$ and inactive (PAL $<1.7)$ groups, QS\% $(\mathrm{p}<0.001)$ and BMI ( $\mathrm{p}=0.02$ ) were the only factors assessed found to differ significantly between the 2 groups (Table 4).

The results of the univariable analysis examining the relationship between PAL and clinical measures are shown in Table 3 and Figure 1. Both the 6MWD and QS\% were positively associated with PAL $(\mathrm{p}<0.1)$ and BMI negatively associated with PAL $(\mathrm{p}<0.1)$. Multivariable results are shown in Table 5 with greater QS\% identified as the only measure independently associated with increased PAL.

The results of the univariable analysis examining the relationship between average daily time spent $\geq 3$ METs and clinical measures are shown in Table 6 and Figure 1. In univariable analysis, 6MWD and QS\% were found to be positively associated with average daily time spent $\geq 3$ METs $(p<0.1)$ and age and BMI were found to be negatively associated with average daily time spent $\geq 3$ METs $(\mathrm{p}<0.1)$. In multivariable analysis greater $\mathrm{QS} \%$ and lower $\mathrm{BMI}$ were identified as the only measures independently associated with increased average daily time spent $\geq 3$ METs (Table 6).

On inspection of the different PAL categories, as PAL increased, average daily time spent at least moderately active ( $\geq 3$ METs) and QS\% increased and BMI decreased (Table 3 \& Figure 2). The active group (PAL $\geq 1.70$ ) were extremely active (mean PAL for this group $2.27 \pm$ 0.41) (Table 3 \& Figure 2). 


\section{DISCUSSION}

This study provides baseline information on the levels of PA of a broad range of adult HTx recipients. Our sample had similar numbers of subjects from each of the 3 PAL and BMI categories. The majority of participants were male $(73.3 \%)$, which is consistent with our hospital HTx cohort where $82 \%$ are male. The mean PAL for the whole group was greater than $1.60(\mathrm{PAL}=1.66 \pm 0.47)$ suggesting that on average, HTx recipients are sedentary to moderately active. However, closer inspection of the data suggests that the majority of participants $(71 \%)$ were essentially inactive $(\mathrm{PAL}<1.70)$ and that $33 \%$ were extremely inactive with a PAL of $<1.40$. The average PAL for the group was increased due to the small number of HTx recipients who were highly active. This active group ( $\mathrm{PAL} \geq 1.70)$ were in some instances extremely active with many exceeding the recommended guidelines of at least 150 minutes of moderate PA per week. Indeed the mean daily time spent moderately active ( $\geq 3$ METs) for this group was $347 \pm 134$ minutes.

Our results reflect the level of PA recorded in the general adult population in western countries (PAL 1.60-1.75) ${ }^{33}$ and reinforce the findings of previous smaller studies post-HTx. A similar amount of daily time spent $\geq 3$ METs was recorded using the SWA in 12 HTx recipients at 1 year post-HTx ${ }^{38}$, sedentary behaviour was observed using mean activity counts from an accelerometer (Actiwatch 2) and self-reporting in 27 women post-HTx(5.2 \pm 4.4years $)^{39}$ and PA levels of 47 HTx recipients $(4.8 \pm 3.0$ years $)$ was reported to be in the low to moderate range of EE assessed using a questionnaire ${ }^{40}$. In contrast, Gustaw et al (2017) in a study assessing PA levels in 113 adult post solid organ transplant recipients via an internetbased questionnaire found adult HTx recipients(24\%; $\mathrm{n}=27)$ were engaged in above limited levels of PA, but stated that this difference may be due to the use of a voluntary convenience sample and participant characteristics ${ }^{54}$. 
Not surprisingly, we found higher QS\% to be independently associated with higher PAL and more average daily time spent at least moderately active $(\geq 3 \mathrm{METs})$. We are unable to determine if the higher QS\% is causally related to increased PAL or vice versa, ie, if PAL results in higher QS\%. We can report that our results are similar to those previously described post-LTx with Langer et al (2009) reporting better quadriceps muscle strength related to higher levels of daily $\mathrm{PA}^{42}$ and Walsh et al (2013) reporting improvements in exercise capacity (measured by 6MWD) largely explained by improvements in QS\% and by recipients' pre-LTx exercise capacity ${ }^{52}$.

We did find an inverse and independent association between BMI and average daily time spent $\geq 3$ METs, suggesting that those with a lower BMI were potentially more active and physically fit. This would appear counter to the 'obesity paradox for HF' (which suggests that higher body weight individuals with HF have better exercise capacity and better chances of survival). Our results are however consistent with the smaller study by Evangelista et al (2005), who also reported an inverse relationship between PA and obesity in 27 women post$\operatorname{HTx}(5.2 \pm 4.4$ years $)$, with more obese individuals being less active ${ }^{39}$.

Interestingly, greater 6MWD (measure of exercise capacity) although found to be related to increased PAL and average daily time spent $\geq 3$ METs was not found to be independently associated with either PAL or time spent $\geq 3$ METs in our HTx sample. This may reflect the fact that 6MWD is an index of exercise capacity and may not necessarily be directly related to PA. Whilst individuals may have the capacity to perform exercise, they may choose not to for a variety of reasons such as motivation and comorbidities, and hence have a low PAL. 
Unfortunately we did not assess quality of life (QoL). Poor QoL may be a reflection of mental health or physical inability and may be more predictive of PA than other physical measures. Assessment of this should be included in future studies. Although the majority of participants were classified as extremely inactive or sedentary they may have been content with their level of physical function. Contrary to our hypothesis, time post-HTx and age were not found to be associated with estimates of PA nor was type of cardiomyopathy.

Other limitations of this study should be acknowledged. First, the design is explorative and cross sectional weakening the strength of conclusions, and due to the very nature of the study, we would have had a survivor bias in our sampling. It is possible and likely that PA may change over time, and those who previously led an active lifestyle may be more likely to be more active post-HTx. Those with a longer history of HF may be less PA post-HTx due to having a longer period to become accustomed to being less PA. Current and previous psychological and behavioural factors that may impact levels of PA such as interests, motivation, anxiety and depression were not investigated either. Diastolic dysfunction was not assessed which may impact PAL in those with preserved LVEF.

Medication regimes may impact PA levels, and although no relationship was found between corticosteroid use at time of assessment and PAL, we were not able to accurately determine the relationship between immunosuppression load post-HTx and PA as time post-HTx varied greatly in our dataset. One may argue that time post-HTx could be a potential surrogate for immunosuppression load (ie, longer period post-HTx would likely have greater immunosuppression load). We also acknowledge as a potential limitation not acquiring information on the role of complications or change in weight in the first year post-HTx and 
eventual outcomes due to 9 participants receiving HTx away from our site and 7 participants being $<12$ months post-HTx at time of assessment.

Although participants were mean $9.2 \pm 7.0$ years post-HTx, participation in a post-HTx cardiac rehabilitation (CR) program and current involvement in a formal exercise program were not recorded. However, early exercise rehabilitation is commenced routinely post-HTx with the aim to mobilize recipients as early as possible postextubation. Daily walking and lower-limb strengthening exercises (sit to stand, squats and stair climbing) are commenced and gradually progressed during the initial hospital admission. On discharge from hospital, all recipients are referred to their local outpatient $\mathrm{CR}$ program if there is one available. Individualised home exercise programs consisting of walking and lower-limb strengthening exercises are also provided which are monitored and progressed during regular outpatient consultations. As all participants were $\geq 6$ months post-HTx it is unlikely that any participants were actively enrolled in a CR program at the time of assessment. A recent retrospective review by Rosenbaum et al (2016) reported an association between increased participation in early $\mathrm{CR}$ and long-term survival in HTx recipients, while adjusting for baseline debility, with attendance early post-HTx corresponding to approximately $10 \%$ decreased risk of death while controlling for baseline 6MWT results, rejection episodes (early graft failure) and corticosteroid dose $\mathrm{e}^{22}$.

We can conclude that management post-HTx is complex. The degree of observed sedentary behaviour post-HTx is surprising, highlighting the need to actively intervene on PA as the majority of participants assessed were not reaching levels of PA recommended for health benefits. Quadriceps muscle strength corrected for body weight and body mass index were the only factors found to be independently associated with estimates of physical activity 
(PAL and average daily time spent at least moderately active ( $\geq 3$ METs)). By encouraging increased levels of PA and by targeting muscle strength recovery in this patient population we may improve long-term health outcomes. Further quality trials are required to demonstrate the long-term benefits of regular PA and investigate ways of increasing adherence to PA post-HTx. 


\section{REFERENCES}

1. Mehra MR, Canter CE, Hannan MM, et al. The 2016 International Society for Heart Lung Transplantation listing criteria for heart transplantation: A 10-year update. $J$ Heart Lung Transplant. 2016;35:1-23.

2. Stehlik J, Edwards LB, Kucheryavaya AY, et al. The Registry of the International Society for Heart and Lung Transplantation: twenty-seventh official adult heart transplant report--2010. J Heart Lung Transplant. 2010;29:1089-1103.

3. Christie JD. The Registry of the International Society for Heart and Lung Transplantation: 29th Adult Lung and Heart-Lung Transplant Report -- 2012. J Heart Lung Transplant. 2012;31:1073-1086.

4. Lund LH, Edwards LB, Kucheryavaya AY, et al. The Registry of the International Society for Heart and Lung Transplantation: Thirtieth Official Adult Heart Transplant Report--2013; focus theme: age. J Heart Lung Transplant. 2013;32:951-964.

5. Milaniak I, Przybylowski P, Wierzbicki K, Sadowski J. Post-Transplantation Body Mass Index in Heart Transplant Recipients: Determinants and Consequences. Transplant Proc. 2014;46:2844-2847.

6. Williams JJ, Lund LH, LaManca J, et al. Excessive weight gain in cardiac transplant recipients. J Heart Lung Transplant. 2006;25:36-41.

7. Russo MJ, Hong KN, Davies RR, et al. The Effect of Body Mass Index on Survival Following Heart Transplantation: Do Outcomes Support Consensus Guidelines? Ann Surg. 2010;251:144-152.

8. Grady KL, Naftel D, Pamboukian SV, et al. Post-operative obesity and cachexia are risk factors for morbidity and mortality after heart transplant: multi-institutional study of post-operative weight change. J Heart Lung Transplant. 2005;24:1424-1430. 
9. Jalowiec A, Anne J, Kathleen LG, Connie W-W. Clinical outcomes in overweight heart transplant recipients. Heart Lung. 2016;45:298.

10. Haskell WL. Physical Activity and Public Health. Updated Recommendation for Adults From the American College of Sports Medicine and the American Heart Association. Circulation. 2007;116:1081-1093.

11. Hu G, Tuomilehto J, Silventoinen K, Barengo NC, Peltonen M, Jousilahti P. The effects of physical activity and body mass index on cardiovascular, cancer and allcause mortality among 47212 middle-aged Finnish men and women. Int J Obes. 2005;29:894.

12. Kokkinos $\mathrm{P}$, Sheriff $\mathrm{H}$, Kheirbek R. Physical inactivity and mortality risk. Cardiol Res Pract. 2011;2011:924945.

13. Blair SN, Blair SN, Cheng Y, Scott Holder J. Is physical activity or physical fitness more important in defining health benefits? Med Sci Sports Exerc. 2001;33:S379S399.

14. Maessen MF, Verbeek AL, Bakker EA, Thompson P, Hopman MT, Eijsvogels TM. Lifelong Exercise Patterns and Cardiovascular Health. Mayo Clin Proc. 2016;91:745754.

15. Thompson PD, Buchner D, Piña IL, et al. Exercise and Physical Activity in the Prevention and Treatment of Atherosclerotic Cardiovascular Disease. Circulation. 2003;107:3109-3116.

16. Di Pietro L, Dziura J, Blair SN. Estimated change in physical activity level (PAL) and prediction of 5-year weight change in men: the Aerobics Center Longitudinal Study. Int J Obes. 2004;28:1541.

17. Marconi C, Marzorati M. Exercise after heart transplantation. Eur J Appl Physiol. 2003;90:250-259. 
18. Rustad LA, Nytroen K, Amundsen BH, Gullestad L, Aakhus S. One year of highintensity interval training improves exercise capacity, but not left ventricular function in stable heart transplant recipients: a randomised controlled trial. Eur J Prev Cardiol. 2014;21:181-191.

19. Gullestad L, Myers J, Edvardsen T, Kjekshus J, Geiran O, Simonsen S. Predictors of exercise capacity and the impact of angiographic coronary artery disease in heart transplant recipients. ACC Curr J Rev. 2004;13:36.

20. Squires RW. Exercise therapy for cardiac transplant recipients. Prog Cardiovasc Dis. 2011;53:429-436.

21. Anderson L, Anderson L, Nguyen TT, Dall CH, Burgess L. Exercise-based cardiac rehabilitation in heart transplant recipients. Cochrane Database Syst Rev. 2017;4:CD012264.

22. Rosenbaum AN, Kremers WK, Schirger JA, et al. Association between early cardiac rehabilitation and long-term survival in cardiac transplant recipients. Mayo Clin Proc. 2016;91:149.

23. Kavanagh T. Exercise rehabilitation in cardiac transplantation patients: a comprehensive review. Eura Medicophys. 2005;41:67-74.

24. Kobashigawa JA, Leaf DA, Lee N, et al. A Controlled Trial of Exercise Rehabilitation after Heart Transplantation. N Engl J Med. 1999;340:272-277.

25. Lampert E, Mettauer B, Hoppeler H, Charloux A, Charpentier A, Lonsdorfer J. Skeletal muscle response to short endurance training in heart transplant recipients. $J$ Am Coll Cardiol. 1998;32:420-426.

26. Braith RW, Magyari PM, Pierce GL, et al. Effect of resistance exercise on skeletal muscle myopathy in heart transplant recipients. Am J Cardiol. 2005;95:1192-1198. 
27. Wever-Pinzon O, Edwards LB, Taylor DO, Kfoury AG. Association of recipient age and causes of heart transplant mortality: Implications for personalization of posttransplant management—An analysis of the International Society for Heart and Lung Transplantation Registry. J Heart Lung Transplant. 2017;36:407-417.

28. Chodzko-Zajko WJ, Proctor DN, Fiatarone Singh MA, et al. Exercise and Physical Activity for Older Adults. Med Sci Sports Exerc. 2009;41:1510-1530.

29. Spruit MA, Singh SJ, Garvey C, et al. An official American Thoracic Society/European Respiratory Society statement: key concepts and advances in pulmonary rehabilitation. Am J Respir Crit Care Med. 2013;188:e13-64.

30. Seefeldt V, Malina RM, Clark MA. Factors Affecting Levels of Physical Activity in Adults. Sports Med. 2002;32:143-168.

31. Sewell L, Singh SJ, Williams JE, Morgan MD. Seasonal variations affect physical activity and pulmonary rehabilitation outcomes. J Cardiopulm Rehabil Prev. 2010;30:329-333.

32. Pitta F, Breyer M-K, Hernandes NA, et al. Comparison of daily physical activity between COPD patients from Central Europe and South America. Respir Med. 2009;103:421-426.

33. Black AE, Coward WA, Cole TJ, Prentice AM. Human energy expenditure in affluent societies: an analysis of 574 doubly-labelled water measurements. Eur J Clin Nutr. 1996;50:72-92.

34. Watz H, Waschki B, Boehme C, Claussen M, Meyer $\mathrm{T}$, Magnussen $\mathrm{H}$. Extrapulmonary effects of chronic obstructive pulmonary disease on physical activity: a cross-sectional study. Am J Respir Crit Care Med. 2008;177:743-751.

35. Hunter GR, Larson-Meyer DE, Sirikul B, Newcomer BR. Muscle metabolic function and free-living physical activity. J Appl Physiol (1985). 2006;101:1256-61. 
36. Manini TM, Everhart JE, Colbert LH, Harris TB, Schoeller DA. Daily Activity Energy Expenditure and Mortality Among Older Adults. JAMA. 2006;296:171-179.

37. Aune D, Norat T, Leitzmann M, Tonstad S, Vatten LJ. Physical activity and the risk of type 2 diabetes: a systematic review and dose-response meta-analysis. Eur $J$ Epidemiol. 2015;30:529-542.

38. Jakovljevic DG, McDiarmid A, Hallsworth K, et al. Effect of left ventricular assist device implantation and heart transplantation on habitual physical activity and quality of life. Am J Cardiol. 2014;114:88-93.

39. Evangelista LS, Dracup K, Doering L, Moser DK, Kobashigawa J. Physical activity patterns in heart transplant women. $J$ Cardiovasc Nurs. 2005;20:334-339.

40. Myers J, Gullestad L, Bellin D, Ross H, Vagelos R, Fowler M. Physical activity patterns and exercise performance in cardiac transplant recipients. $J$ Cardiopulm Rehabil. 2003;23:100-106.

41. Langer D, Burtin C, Schepers L, et al. Exercise Training After Lung Transplantation Improves Participation in Daily Activity: A Randomized Controlled Trial. Am J Transplant. 2012;12:1584-1592.

42. Langer D, Gosselink R, Pitta F, et al. Physical Activity in Daily Life 1 Year After Lung Transplantation. J Heart Lung Transplant. 2009;28:572-578.

43. Bossenbroek L, den Ouden ME, de Greef MH, Douma WR, Ten Hacken NH, van der Bij W. Determinants of overweight and obesity in lung transplant recipients. Respiration. 2011;82:28-35.

44. Bossenbroek L, ten Hacken NHT, van der Bij W, Verschuuren EAM, Koëter GH, de Greef MHG. Cross-sectional assessment of daily physical activity in chronic obstructive pulmonary disease lung transplant patients. J Heart Lung Transplant. 2009;28:149-155. 
45. Watz H, Waschki B, Meyer T, Magnussen H. Physical activity in patients with COPD. Eur Respir J. 2009;33:262-272.

46. Johannsen DL, Calabro MA, Stewart J, Franke W, Rood JC, Welk GJ. Accuracy of armband monitors for measuring daily energy expenditure in healthy adults. Med Sci Sports Exerc. 2010;42:2134-2140.

47. St-Onge M, Mignault D, Allison DB, Rabasa-Lhoret R. Evaluation of a portable device to measure daily energy expenditure in free-living adults. Am J Clin Nutr. 2007;85:742-749.

48. Patel SA, Benzo RP, Slivka WA, Sciurba FC. Activity monitoring and energy expenditure in COPD patients: a validation study. COPD. 2007;4:107-112.

49. Furlanetto KC, Bisca GW, Oldemberg N, et al. Step counting and energy expenditure estimation in patients with chronic obstructive pulmonary disease and healthy elderly: accuracy of 2 motion sensors. Arch Phys Med Rehabil. 2010;91:261-267.

50. O'Shea SD, Taylor NF, Paratz JD. Measuring muscle strength for people with chronic obstructive pulmonary disease: retest reliability of hand-held dynamometry. Arch Phys Med Rehabil. 2007;88:32-36.

51. Walsh JR, Morris NR, McKeough ZJ, Yerkovich ST, Paratz JD. A simple clinical measure of quadriceps muscle strength identifies responders to pulmonary rehabilitation. Pulm Med. 2014;2014:782702.

52. Walsh JR, Chambers DC, Davis RJ, et al. Impaired exercise capacity after lung transplantation is related to delayed recovery of muscle strength. Clin Transplant. 2013;27:E504-511.

53. ATS statement: guidelines for the six-minute walk test. Am J Respir Crit Care Med. 2002;166:111-117. 
54. Gustaw T, Schoo E, Barbalinardo C, et al. Physical activity in solid organ transplant recipients: Participation, predictors, barriers, and facilitators. Clin Transplant. 2017;31:e12929. 

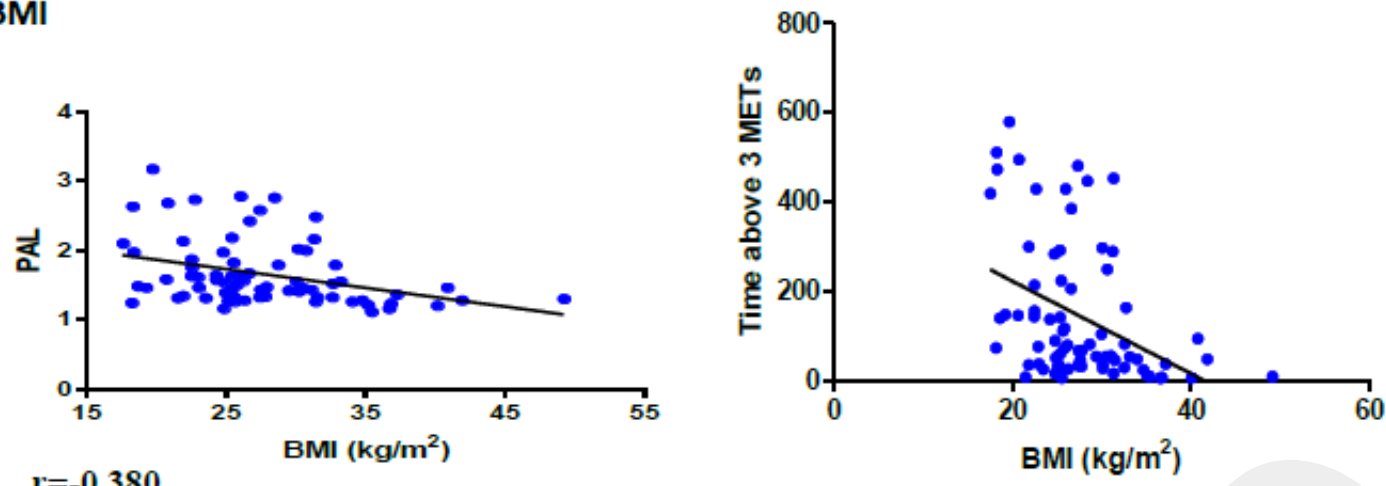

$r=-0.380$

$\mathrm{p}=0.001$

$r=-0.420$
$p<0.001$

6MWD
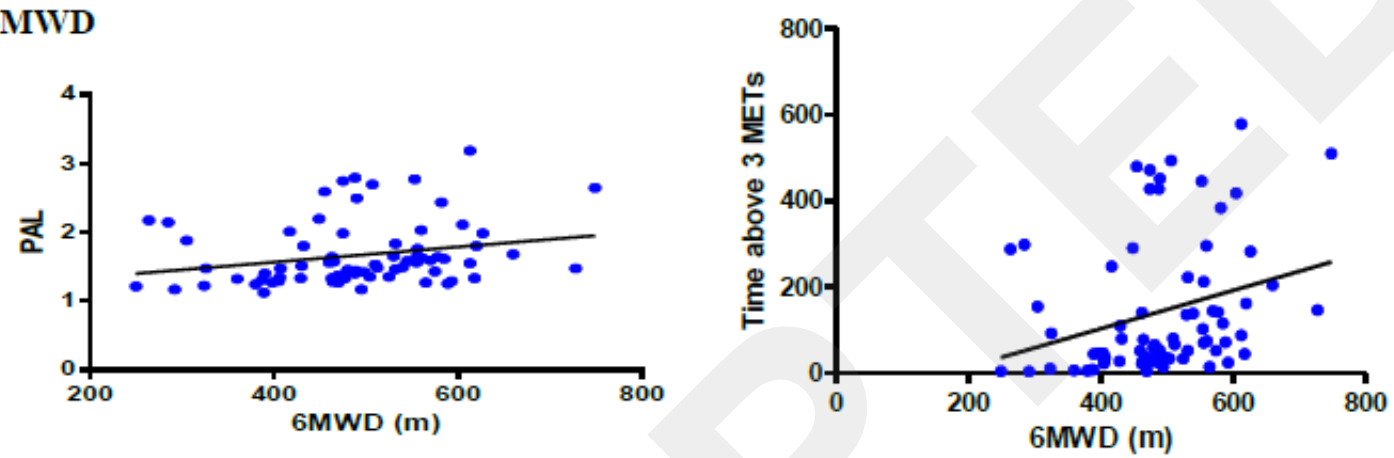

$\mathrm{r}=\mathbf{0 . 3 3 0}$

$\mathrm{p}=0.004$

$\mathrm{r}=\mathbf{0 . 4 0 0}$

$\mathrm{p}<0.001$

Quadriceps muscle strength (QS\%)
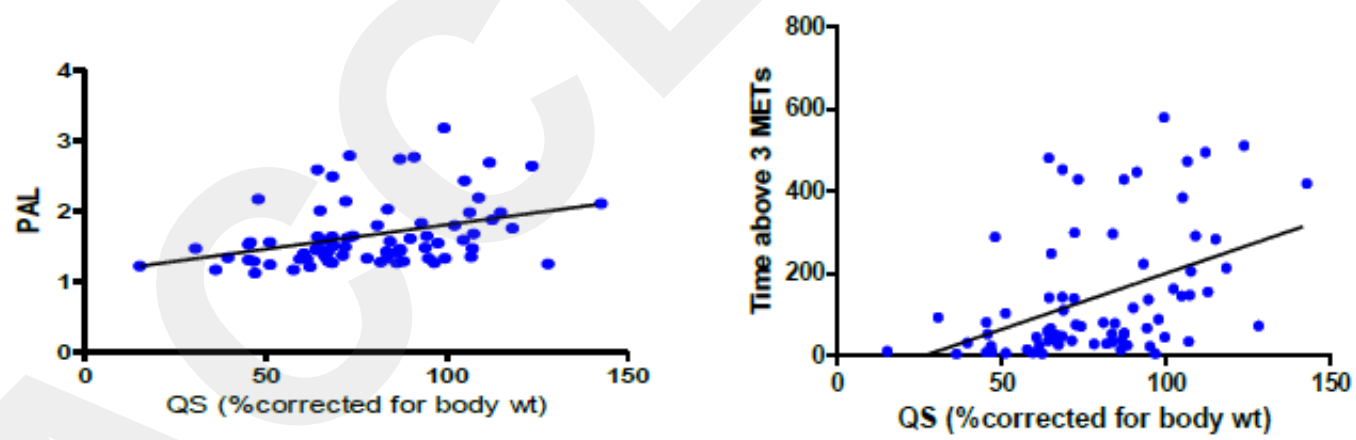

$r=0.420$

$\mathrm{p}=0.001$

$r=0.490$

$\mathrm{p}<0.001$ 
Figure 1

BMI
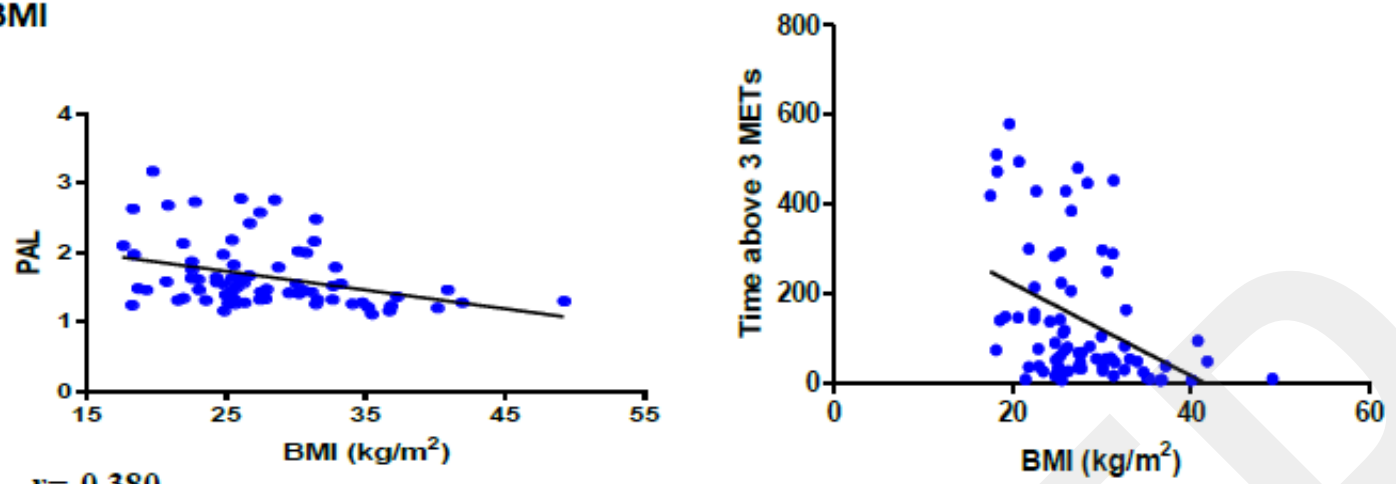

$r=-0.380$
$p=0.001$

$r=-0.420$

$\mathrm{p}<0.001$

6MWD
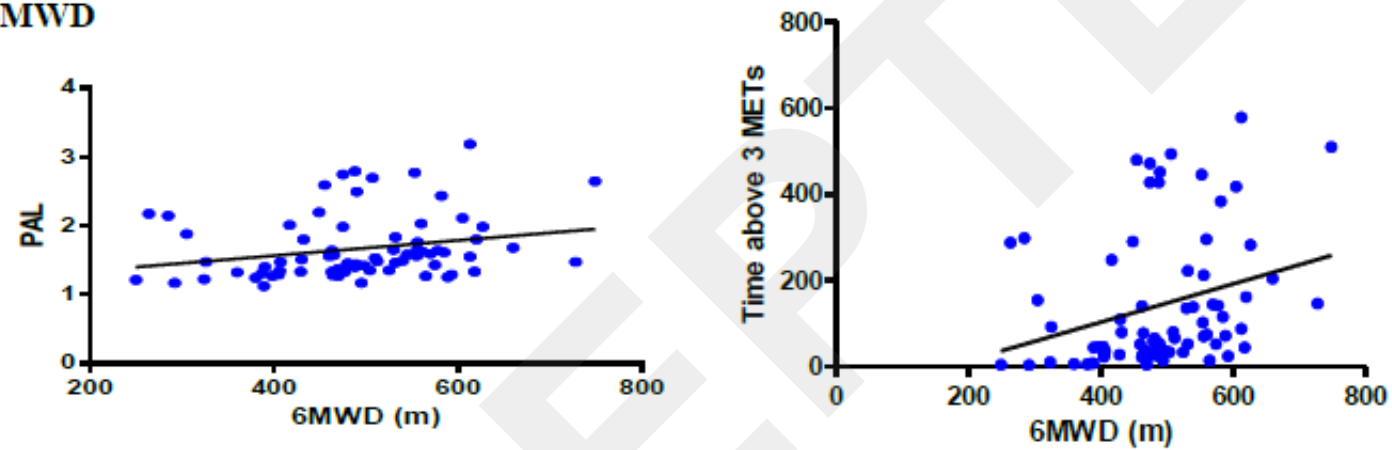

$r=0.330$

$r=0.400$

$\mathrm{p}<0.001$

Quadriceps muscle strength (QS\%)
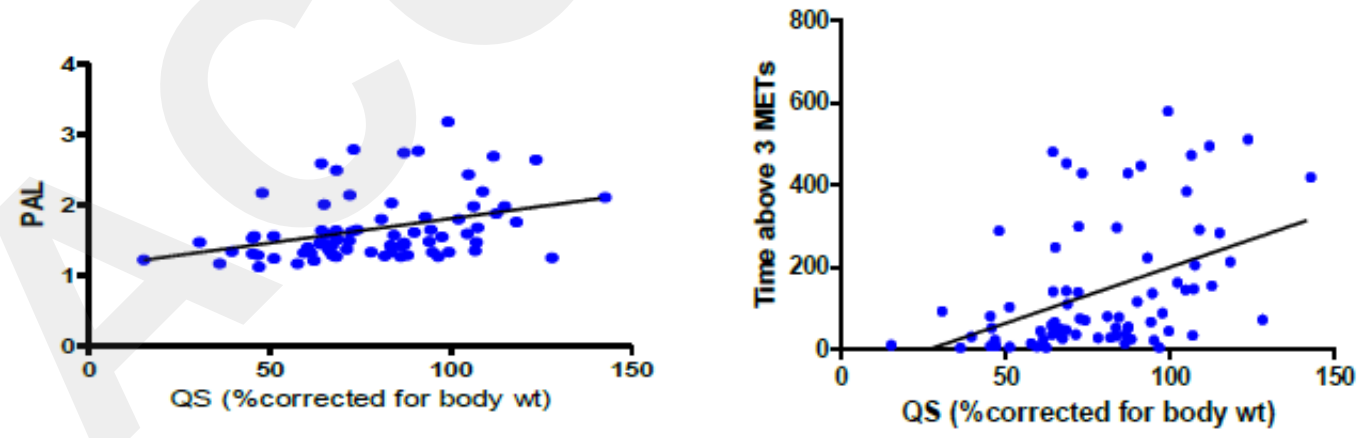

$r=0.420$

$\mathrm{p}=0.001$

$r=0.490$

$\mathrm{p}<0.001$ 
Figure 2

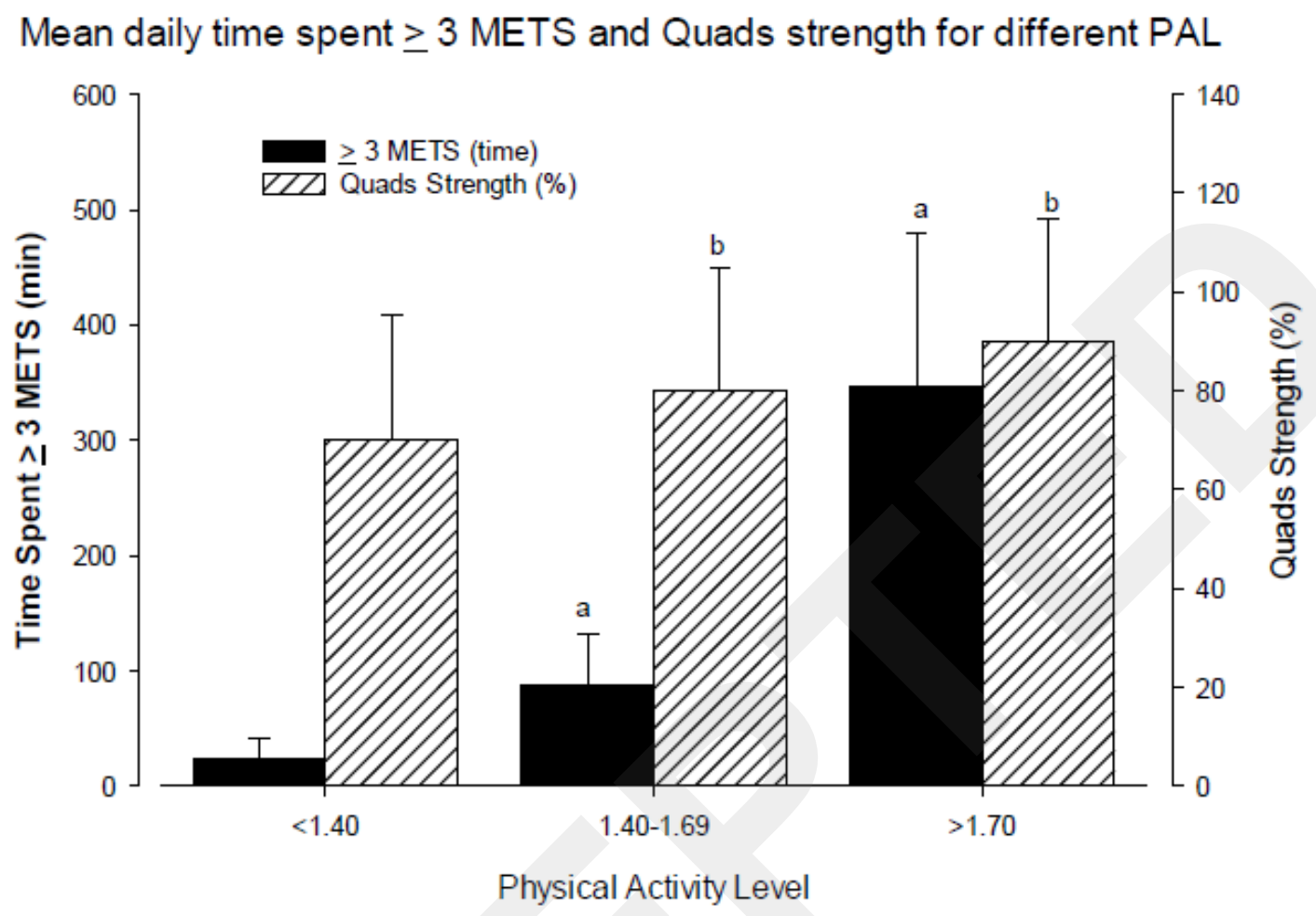


Table 1: Demographics

\begin{tabular}{|l|c|}
\hline Demographics (n=75) & \\
\hline Age (years) & $56 \pm 14$ \\
\hline Female (n, \%) & $20,26.7$ \\
\hline Body Mass $(\mathrm{kg})$ & $82.6 \pm 19.3$ \\
\hline Height $(\mathrm{cm})$ & $172.0 \pm 8.7$ \\
\hline Body mass index $\left(\mathrm{kg} / \mathrm{m}^{2}\right)$ & $27.7 \pm 6.1$ \\
\hline Time posttransplant (years) & $9.2 \pm 7.0$ \\
\hline Left ventricular ejection fraction (\%) & $60.8 \pm 7.2$ \\
\hline 6MWD (m) & $488 \pm 100$ \\
\hline $\begin{array}{l}\text { Quadriceps strength corrected } \\
\text { for body weight (QS\%) }\end{array}$ & $78.8 \pm 24.6$ \\
\hline
\end{tabular}

Table 1: Demographic data, exercise capacity (6MWD) and quadriceps strength corrected for body weight (QS\%). Categorical data presented as mean $\pm S D$. 
Table 2: Comparison of corticosteroid use of active $(\mathrm{PAL} \geq 1.7)$ and inactive $(\mathrm{PAL}<1.7)$ groups

\begin{tabular}{|c|c|c|c|}
\hline $\begin{array}{c}\text { Corticosteroid (prednisolone) } \\
\text { use at time of assessment }\end{array}$ & $\begin{array}{c}\text { Active group } \\
\text { (PAL } \geq \mathbf{1 . 7})\end{array}$ & $\begin{array}{c}\text { Inactive group } \\
\text { (PAL<1.7) }\end{array}$ & Total \\
\hline Yes & 5 & 21 & 26 \\
\hline No & 17 & 32 & 49 \\
\hline Total & 22 & 53 & 75 \\
\hline
\end{tabular}

Table 2: Corticosteroid (prednisolone) use at time of assessment. 
Table 3: Physical activity parameters

\begin{tabular}{|l|c|c|c|c|c|c|}
\hline $\begin{array}{l}\text { Outcomes } \\
\text { (n=75) }\end{array}$ & \% & PAL & $\begin{array}{c}\text { Mean daily } \\
\text { time spent } \\
\geq 3 \text { METs } \\
\text { (mins) }\end{array}$ & BMI & Age & QS\% \\
\hline $\begin{array}{l}\text { TOTAL } \\
\text { GROUP } \\
(\mathbf{n = 7 5 )}\end{array}$ & 100 & $1.66 \pm 0.47$ & $143 \pm 155$ & $27.7 \pm 6.1$ & $56 \pm 14$ & $78.8 \pm 24.6$ \\
\hline $\begin{array}{l}\text { Extremely } \\
\text { inactive } \\
(<1.40) \\
(\mathrm{n}=25)\end{array}$ & 33.3 & $1.28 \pm 0.06$ & $24.56 \pm 16.95$ & $30.45 \pm 7.18$ & $57.64 \pm 13.64$ & $69.57 \pm 25.35$ \\
\hline $\begin{array}{l}\text { Sedentary } \\
(1.40-1.69) \\
(\mathrm{n}=28)\end{array}$ & 37.3 & $1.52 \pm 0.09$ & $88.14 \pm 44.00$ & $26.96 \pm 4.78$ & $54.19 \pm 13.18$ & $76.04 \pm 19.91$ \\
\hline $\begin{array}{l}\text { Active } \\
(\geq 1.70) \\
(\mathrm{n}=22)\end{array}$ & 29.3 & $2.27 \pm 0.41$ & $346.77 \pm 133.95$ & $25.15 \pm 4.64$ & $55.54 \pm 16.99$ & $94.21 \pm 23.17$ \\
\hline
\end{tabular}

Table 3: Physical activity level (PAL), mean daily time spent $\geq 3$ METs, BMI, age and QS\%.

Categorical data presented as mean $\pm S D$. 
Table 4: Characteristics of active $(\mathrm{PAL} \geq 1.7)$ and inactive $(\mathrm{PAL}<1.7)$ groups

\begin{tabular}{|l|c|c|c|}
\hline Characteristics & Active group (n=22) & Inactive group (n=54) & $\mathbf{p}$ \\
\hline Age (years) & $55.5 \pm 17.0$ & $56 \pm 13.4$ & 0.90 \\
\hline Female (n, \%) & 7,32 & 13,24 & 0.57 \\
\hline BMI (kg/m $\mathbf{m}^{\mathbf{2}}$ & $\mathbf{2 5 . 2} \pm \mathbf{4 . 6}$ & $\mathbf{2 8 . 7} \pm \mathbf{6 . 3}$ & $\mathbf{0 . 0 2}$ \\
\hline Time posttransplant (years) & $9.9 \pm 7.2$ & $8.7 \pm 7.0$ & 0.52 \\
\hline Left ventricular ejection fraction (\%) & $60.3 \pm 6.2$ & $61 \pm 7.5$ & 0.72 \\
\hline 6MWD (m) & $501.8 \pm 117.8$ & $483 \pm 93.4$ & 0.47 \\
\hline $\begin{array}{l}\text { Quadriceps strength corrected for } \\
\text { body weight (QS\%) }\end{array}$ & $\mathbf{9 4 . 2} \pm \mathbf{2 3 . 2}$ & $\mathbf{7 2 . 5} \pm \mathbf{2 2 . 4}$ & $<\mathbf{0 . 0 0 1}$ \\
\hline Taking corticosteroid (n, \%) & $5,22.7$ & $21,39.6$ & 0.19 \\
\hline
\end{tabular}

Table 4: Demographic data, exercise capacity (6MWD), quadriceps strength corrected for body weight (QS\%) and corticosteroid use at time of assessment. Categorical data presented as mean \pm $S D$. 
Table 5: Univariable \& multivariable analysis: Predictors of PAL

\begin{tabular}{|l|c|c|c|}
\hline Univariable: PAL & B & $\mathbf{9 5 \%}$ CI & p \\
\hline BMI & -0.015 & $-0.024--0.007$ & 0.001 \\
\hline 6MWD & 0.001 & $0.000-0.012$ & 0.024 \\
\hline QS\% & 0.004 & $0.002-0.006$ & $<0.001$ \\
\hline Multivariable: PAL & $\mathbf{B}$ & $\mathbf{9 5 \%}$ CI & $\mathbf{p}$ \\
\hline QS\% & 0.004 & $0.002-0.006$ & 0.001 \\
\hline
\end{tabular}

Table 5: Physical activity level (PAL): dependent variable. Independent variables evaluated: Participants' age, sex, time post-HTx, body mass index (BMI), reason for HTx, 6-minute walk distance (6MWD) and quadriceps muscle strength corrected for body weight (QS\%). 
Table 6: Univariable \& multivariable analysis: Predictors of mean daily time spent $\geq 3 \mathrm{METs}$

\begin{tabular}{|l|c|c|c|}
\hline Univariable: Time spent $\geq$ 3METs & B & 95\% CI & p \\
\hline Age & -0.021 & $-0.041--0.001$ & 0.042 \\
\hline BMI & -0.102 & $-0.145--0.059$ & $<0.001$ \\
\hline 6MWD & 0.005 & $0.003-0.008$ & $<0.001$ \\
\hline QS\% & 0.026 & $0.015-0.037$ & $<0.001$ \\
\hline Multivariable: Time spent $\geq \mathbf{3 M E T s}$ & $\mathbf{B}$ & $\mathbf{9 5 \%}$ CI & $\mathbf{p}$ \\
\hline QS\% & 0.016 & $0.003-0.029$ & 0.014 \\
\hline BMI & -0.063 & $-0.115--0.010$ & 0.021 \\
\hline
\end{tabular}

Table 6: Mean daily time spent $\geq 3 M E T s$ : dependent variable. Independent variables evaluated: Participants' age, sex, time post-HTx, body mass index (BMI), reason for HTx, 6-minute walk distance (6MWD) and quadriceps muscle strength corrected for body weight (QS\%). 